\title{
Exon Skipping-Mediated Dystrophin Reading Frame Restoration for Small Mutations
}

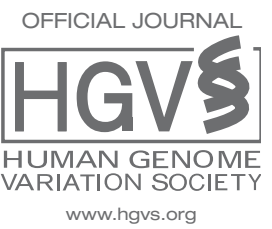

\author{
Pietro Spitali, ${ }^{1 \dagger}$ Paola Rimessi, ${ }^{1 \dagger}$ Marina Fabris, ${ }^{1}$ Daniela Perrone ${ }^{2,3}$ Sofia Falzarano, ${ }^{1}$ Matteo Bovolenta, ${ }^{1}$ Cecilia Trabanelli, \\ Lara Mari, ${ }^{2}$ Elena Bassi, ${ }^{1}$ Sylvie Tuffery, ${ }^{4}$ Francesca Gualandi, ${ }^{1}$ Nadir M. Maraldi, ${ }^{5}$ Patrizia Sabatelli-Giraud, ${ }^{5}$ \\ Alessandro Medici, ${ }^{3}$ Luciano Merlini, ${ }^{1}$ and Alessandra Ferlini ${ }^{1 *}$ \\ ${ }^{1}$ Department of Experimental and Diagnostic Medicine, Section of Medical Genetics, University of Ferrara, Italy; ${ }^{2}$ Department of Chemistry, \\ University of Ferrara, Ferrara, Italy; ${ }^{3}$ Department of Biology and Evolution, University of Ferrara, Ferrara, Italy; ${ }^{4}$ Université Montpellier 1, Faculté \\ de Médecine and Inserm, U827, Montpellier, F-34000, France; ${ }^{5}$ Institute of Anatomy, University of Bologna and IGM-CNR., Unit of Bologna, \\ c/o IOR, Bologna, Italy
}

Communicated by Christophe Béroud

Received 17 March 2009; accepted revised manuscript 30 June 2009.

Published online 14 July 2009 in Wiley InterScience (www.interscience.wiley.com). DOI 10.1002/humu.21092

ABSTRACT: Exon skipping using antisense oligonucleotides (AONs) has successfully been used to reframe the mRNA in various Duchenne muscular dystrophy patients carrying deletions in the DMD gene. In this study we tested the feasibility of the exon skipping approach for patients with small mutations in in-frame exons. We first identified 54 disease-causing point mutations. We selected five patients with nonsense or frameshifting mutations in exons $10,16,26,33$, and 34 . Wild-type and mutation specific $2^{\prime} \mathrm{OMePS}$ AONs were tested in cell-free splicing assays and in cultured cells derived from the selected patients. The obtained results confirm cell-free splicing assay as an alternative system to test exon skipping propensity when patients' cells are unavailable. In myogenic cells, similar levels of exon skipping were observed for wild-type and mutation specific AONs for exons 16, 26, and 33, whereas for exon 10 and exon 34 the efficacy of the AONs was significantly different. Interestingly, in some cases skipping efficiencies for mutated exons were quite dissimilar when compared with previous reports on the respective wild-type exons. This behavior may be related to the effect of the mutations on exon skipping propensity, and highlights the complexity of identifying optimal AONs for skipping exons with small mutations.

Hum Mutat 30:1527-1534, 2009. (c) 2009 Wiley-Liss, Inc.

KEY WORDS: exon skipping; antisense oligonucleotides; dystrophin; DMD
Additional Supporting Information may be found in the online version of this article. †The first two authors contributed equally to this article.

*Correspondence to: Alessandra Ferlini, Department of Experimental and Diagnostic Medicine, Section of Medical Genetics, University of Ferrara, 7444100 Ferrara, Italy. E-mail: fla@unife.it

\section{Introduction}

Nonsense or frame-shifting mutations in the DMD gene (MIM\# 310377) lead to Duchenne muscular dystrophy (DMD; MIM\# 310200), a severe X-linked neuromuscular disorder due to the complete absence of functional dystrophin protein in males. By contrast, the milder allelic Becker muscular dystrophy (BMD; MIM\# 310376) is caused by in-frame mutations that give rise to a smaller but functional protein [Hoffman et al., 1987; Monaco, 1989]. The interruption or maintenance of the dystrophin reading frame by the gene mutations explains the phenotypic differences observed in approximately $92 \%$ of the BMD/DMD cases [Aartsma-Rus et al., 2006; Koenig et al., 1989]. Although the majority of the mutations in the DMD gene consists of large rearrangements, an increasing number of both nonsense and small frame-shifting mutations has been widely reported, due to the improvement of the diagnostic systems [Bennett et al., 2001; Buzin et al., 2005; Flanigan et al., 2003; Hofstra et al., 2004; Roberts et al., 1993, 1994; Tuffery-Giraud et al., 2004; Whittock et al., 1997]. It is now estimated that point mutations account for nearly $30 \%$ of dystrophin mutations [Aartsma-Rus et al., 2006; Deburgrave et al., 2007].

Due to the approach of mutation-specific clinical trials, dystrophin mutation characterization meanwhile has become mandatory. For instance, only patients carrying nonsense mutations are eligible for participation in the phase I/IIa trial held by PTC Therapeutics, based on a stop codon read-through strategy [Welch et al., 2007]. Another mutation-specific approach is antisenseinduced exon skipping aimed at the reframing of dystrophin transcripts. Among the various antisense types or modifications, the $2^{\prime} \mathrm{OMePS}$ antisense oligonucleotides (AONs) targeting exon 51 have been recently used in a pilot study in four DMD patients with very encouraging results [van Deutekom et al., 2007].

Because the majority of DMD-causing mutations are out-offrame deletions clustered within the two major hotspot regions, the exon skipping approach has been focused on this type of rearrangements. However, it has recently been estimated that, in theory, single and double exon skipping would be applicable to $79 \%$ of deletions, $91 \%$ of small mutations, and $73 \%$ of duplications, amounting to $83 \%$ of all DMD mutations [AartsmaRus et al., 2009]. Indeed, only two nonsense mutations were approached in vitro by 2 'OMePS AONs designed on the wild-type exon sequence [Aartsma-Rus et al., 2003, 2004]. 
Although single exon skipping for small mutations can be applied for mutations lying within in-frame exons only, many patients are still eligible for such a treatment (11\% of all DMD patients; Aartsma-Rus et al., 2009). Therefore, the general aim of this study was to confirm the applicability of single exon skipping to small mutations. We also intended to evaluate whether "universal" or "private," mutation-specific AONs were more effective in inducing the exon skipping of mutated dystrophin exons, effectively impacting the design of future therapies for these patients. It is, in fact, known that small mutations can disrupt exon splicing enhancers (ESEs) and silencers (ESSs), splicing regulatory sequences devoted to the exon recognition process [Cartegni et al., 2003]. Mutations located in ESE and ESS elements may affect splicing. Consequently, a significant proportion of exonic point mutations could exert an effect completely different as would be predicted from the genetic code. Positive and negative regulatory elements play opposite and fundamental roles in a finely tuned exon recognition process [Nielsen et al., 2007], which need to be taken into account when interfering with the splicing process. Because mutations lying within ESEs may strongly affect the splicing process [Solis et al., 2008], we used this double approach to point out this relevant topic.

We have identified 54 unrelated DMD patients carrying small mutations in the DMD gene, of which 32 are novel. Among 24 patients carrying a mutation in an in-frame exon, we selected 5 mutations in 5 different exons to be modulated (listed in Table 1). We induced the specific exon skipping using AONs designed either on the wild-type or on the mutated sequence both in cell-free splicing assays (four exons) and myogenic cell cultures (all five exons). The predictive value of a cell-free splicing assay on exon skipping propensity was assessed to have an alternative assay in case cells from the patients were not available. The AONs design was performed on the basis of well established in silico parameters (sites for SC35 and SF2/ASF binding sites) [Aarstma-Rus et al., 2005, 2008]. This approach follows the concept of targeting exon sequences containing ESEs. It has, in fact, already been established that AONs-mediated exon skipping occurs by blocking the access of the splicing machinery to ESE sites in the exon [Aartsma-Rus et al., 2006]. Exon-internal AONs have proven to be more effective than AONs lying on the canonical splice sites [Aartsma-Rus et al., 2008]. They also reduced the possible risk of off-targeting of nonspecific sequences [Aartsma-Rus et al., 2004].

We showed that skipping efficiencies for certain mutated exons were significantly different from what has been previously reported for the respective wild-type exons. This is the first report describing AONs modulation of dystrophin exons containing point mutations through efficacy comparison of the wild-type versus the mutation-specific "private" AONs.

\section{Material and Methods}

\section{Mutation Analysis}

DNA from 54 unrelated DMD patients was extracted by QIAGEN BioRobot (Universal System 8000) from whole blood, after informed consent and for diagnostic purposes. All these patients were negative for DMD deletions/duplications as assessed by MLPA.

We designed a set of 79 primer pairs for single-exon amplification. Oligonucleotide sequences and amplification conditions are available upon request. The amplicons were sequenced using a 3130 automated sequencer (Applied Biosystem, Bedford, MA)

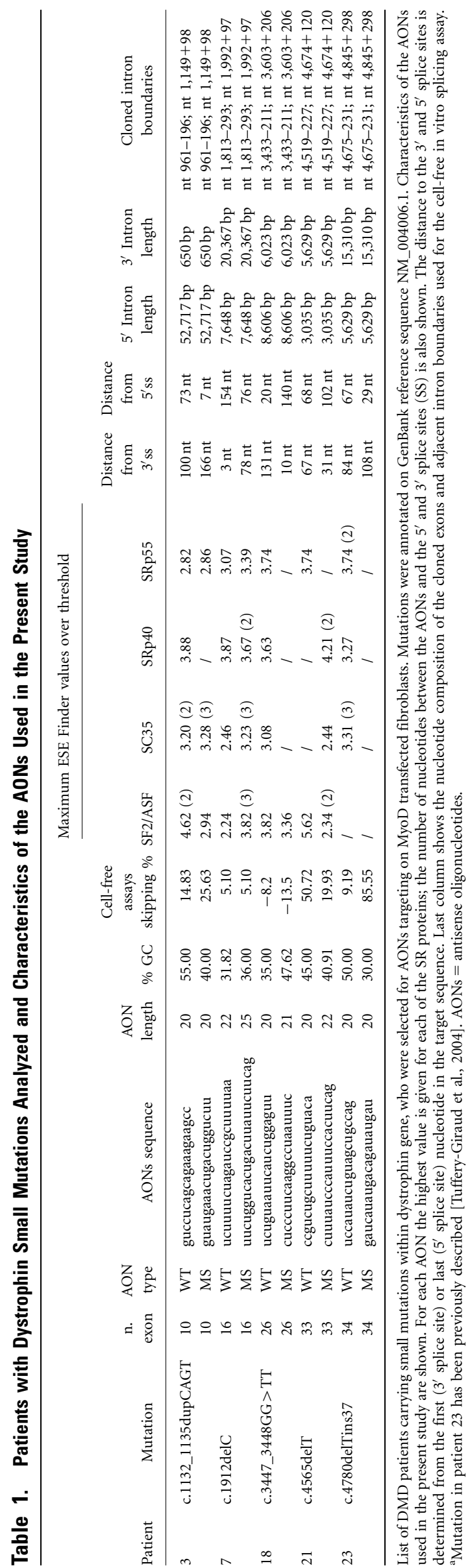


following the manufacturer's recommendations. Supp. Table S1 lists the mutations identified. Mutation numbering system is based on cDNA sequence (GenBank reference sequence NM_004006.1) in which nucleotide numbering reflects cDNA numbering with +1 corresponding to the A of the ATG translation initiation codon in the reference sequence. The initiation codon is codon 1 .

\section{Constructs}

The five selected mutated exons and relative intron boundaries (shown in Table 1) were amplified from the patients' genomic DNAs using in-house protocols (available upon request) and cloned in the previously described pBG vector [Gualandi et al., 2003]. Forward primers for each amplicon were designed upstream of the branch site consensus, whereas reverse primers were located at least 100 nucleotides downstream of the donor splice site. The correct nucleotide composition of all constructs was confirmed by sequencing.

\section{AONs Design and Synthesis}

Wild-type AON design was based on the highest ESE-Finder value [Cartegni et al., 2003) (http://rulai.cshl.edu/cgi-bin/tools/ ESE3/esefinder.cgi process $=$ home) and on the scores for SF2/ ASF and SC35, paying attention to the absolute distance to the $3^{\prime}$ splice site as previously reported [Aartsma-Rus et al., 2005]. AONs sequences, characteristics, and chosen parameters are shown in Table 1.

The AONs were synthesized with a full-length phosphorothioate backbone and 2'-O-methyl modified ribose molecules. Oligonucleotide synthesis was carried out on an $\ddot{\mathrm{AKTA}}{ }^{\mathrm{TM}}$ oligopilot plus 10 DNA/RNA synthesizer (GE Healthcare, Piscataway, NJ) using its trityl-on mode. The sequence was synthesized on a $2-\mu \mathrm{mol}$ scale using Primer Support 200 loaded at $80 \mu \mathrm{mol} / \mathrm{g}$ (Amersham Biosciences, Piscataway, NJ) as previously described [Rimessi et al., 2009]. The final oligonucleotide was dissolved in water and filtered through a short column of Dowex 50WX8 (Na + form, 100-200 mesh) to afford after lyophilization $0.8 \mu \mathrm{mol}(40 \%)$ of target compound. The purity of the full-length desired product was confirmed by MALDI-TOF MS, ${ }^{31} \mathrm{P}-\mathrm{NMR}$ and RP-HPLC analyses.

\section{In Vitro Cell-Free Splicing Assay}

Five in-frame mutated exons cloned into the pBG splicing vector, were transcribed in vitro using T7 RNA polymerase and $\alpha^{32}$-rGTP, and purified from $5 \%$ acrylamide gel to perform in vitro cell-free splicing assays in HeLa nuclear extracts, as previously described [Gualandi et al., 2003]. All the splicing products were reverse transcribed using a High-capacity cDNA Reverse Transcription Kit (Applied Biosystems). cDNAs were amplified using the Ex2/Ex3 oligos designed on the rabbit $\beta$-globin sequences [Gualandi et al., 2003], cloned in the pCRII vector (TA Cloning Invitrogen, Carlsbad, CA), and sequenced on ABI Prism 3130 (Applied Biosystems) to confirm the accuracy of the exon splicing process (amplification conditions and primer sequences are available upon request).

To induce exon skipping in the cell-free in vitro system, $2^{\prime} \mathrm{OMePS}$ AONs were used in concentrations ranging from 100 to $500 \mathrm{nM}$. The incubation lasted 3 hours. To semiquantify the amount of the skipped transcript we performed quantification on images from scanned autoradiographs. The fragments were quantified by Quantity One software (BioRad, Hercules, CA). We compared the amount of spliced products with the total amount product in the presence as well as in the absence of AON. We assumed that the total of the two transcripts (unspliced/ spliced) accounts for $100 \%$.

\section{Myogenic Cell Cultures and AONs Transfection}

Primary human fibroblasts from five patients $(3,7,18,21$, and 23 in Supp. Table S1 and listed also in Table 1) were isolated from skin biopsies (obtained after informed consent for research purposes, Ethical Approval N. 9/2005). Cells were cultivated in high-glucose DMEM (GIBCO, Gaithersburg, MD), supplemented with 20\% fetal bovine serum (FBS; GIBCO) and antibiotic/ antimicotic solution (Sigma, St. Louis, MO). Myogenesis was induced by infection with an Ad5-derived, EA1-deleted adenoviral vector carrying the MyoD gene as previously described [AartsmaRus et al., 2003; Havenga et al., 2002; Roest et al., 1996]. All experiments were carried out using cells with a maximum of four passages. Myotubes obtained after 10-14 days of culture in differentiation medium ( $2 \%$ FBS) were transfected with AONs $(100 \mathrm{nM})$ in the presence of polyethylenimine (ExGen500, MBI Fermentas) $(2 \mu \mathrm{l}$ per $\mu \mathrm{g}$ of $\mathrm{AON})$ as transfection reagent, as previously described [Van Deutekom et al., 2007].

\section{Myogenic Cultures RNA Studies}

Forty-eight hours posttransfection, total RNA was isolated from myotube cultures (RNeasy Kit, Qiagen, Chatsworth, CA) and reverse transcribed into cDNA using random primers and the High-Capacity cDNA Reverse Transcription Kit (Applied Biosystems). RT-PCR was performed on $\beta$-actin to verify cDNA synthesis and on dystrophin (primers sequence available upon request) using primers situated on several flanking exons to exclude spontaneous multi-exon skipping. For exons 26 and 34 a nested PCR was necessary to detect specific exon skipping.

PCRs were performed by five cycles of $94^{\circ} \mathrm{C}(30 \mathrm{sec}), 63^{\circ} \mathrm{C}$ $(30 \mathrm{sec})$, and $72^{\circ} \mathrm{C}(30 \mathrm{sec})$ plus 30 cycles of $94^{\circ} \mathrm{C}(30 \mathrm{sec}), 62^{\circ} \mathrm{C}$ $(30 \mathrm{sec})$, and $72^{\circ} \mathrm{C}(30 \mathrm{sec})$. PCR products were analyzed on $1.5 \%$ agarose gels. All amplified fragments were sequenced on $\mathrm{ABI}$ Prism 3130 (Applied Biosystems). We did not perform semiquantitative densitometric analysis on RT-PCR products, because we adopted the Real-time quantitative assay.

\section{Exon-Specific Real-Time Assay (ESRA)}

To precisely quantify the percentage of exon skipping we set up eight novel ESRAs detecting human dystrophin exons 6, 10, 16, $26,33,34,40$, and 70 . These exons were chosen because they are not involved in spontaneous alternative splicing events in humans. We performed the ESRAs on exons 6, 10, 16, 26, 40, and 70, considered as references, to quantify the amount of dystrophin transcript both in patients' and in control myotubes. In addition, the same ESRAs were used to measure the level of physiological exon skipping and the percentage of induced exon skipping in treated cells compared to untreated cells (internal reference). These data were confirmed using an external reference gene ( $\beta$-actin gene) on cDNA samples from each myotube culture (treated and control cells). All ESRAs are based on TaqMan MGB technology, and have been designed by PrimerExpress Applied Biosystems software (primer and probe sequences are available upon request). The amount of the target sequences in respect to internal references (represented by a nonmutated dystrophin 
exon) and to an appropriate endogenous control ( $\beta$-actin gene) was evaluated by the comparative CT method in respect to the untreated control ( $\Delta \Delta \mathrm{Ct}$ Method) (Applied Biosystems User Bullettin \#2).

\section{Immunofluorescence Analysis}

Treated myotube cultures were grown onto coverslips and fixed in $-20^{\circ} \mathrm{C}$ methanol at 2-6 days posttransfection, depending on the survival rate of the myotubes. Samples were incubated for $30 \mathrm{~min}$ in phosphate-buffered saline (PBS) additioned with $4 \%$ bovine serum albumin. All samples were labeled with a polyclonal antidystrophin antibody (H300 Santa Cruz Biotechnology, Santa Cruz, CA) diluted 1:40, washed with PBS, and revealed with a TRITC-conjugated antirabbit secondary antibody; all samples were double labeled with mouse monoclonal antibodies against desmin or developmental myosin heavy chain (Novocastra Laboratories Ltd., Newcastle upon Tyne, UK), diluted 1:10 and 1:60, respectively. After several washings with PBS, all samples were incubated with FITCconjugated antimouse secondary antibodies (Dako, Carpenteria, $\mathrm{CA})$. The slides were mounted with antifade mounting medium (Molecular Probes, Eugene, OR) and analyzed using Nikon Eclipse 80i fluorescence microscope.

\section{Results}

\section{Small Mutations Identification}

A series of 54 patients with no deletions or duplications were screened for small mutations. Identified mutations are reported in Supp. Table S1. Among the 54 mutations, 32 are novel. The mutation in patient 23 was previously described [TufferyGiraud et al., 2004] and has been included in Table 1 (and Supp. Table S1), as the fibroblasts of this patient were used for AON modulation.

Pathogenic mutations were identified for each patient. Mutations include splicing, frame shifting, and nonsense mutations, and 24 DMD patients show small mutations in 16 different in-frame exons. To confirm the applicability of exon skipping for small mutations occurring within in-frame exons we selected five patients to be investigated (number 3 [NM_004006.1:c.1132_1135dup in exon 10], 7 [NM_004006.1:c.1912_1912delC in exon 16], 18 [NM_004006.1:c. 3447_3448delGGinsTT in exon 26], 21 [NM_004006.1:c.4565delT in exon 33], and 23 [NM_004006.1:c.4780delTins37 in exon 34]).

\section{AONs Design}

For each exon we designed AONs specific for the mutation (mutation specific, msAONs) and AONs not covering the mutations (wild-type, wtAONs) (see Table 1). AONs targeting exons 16 and 26 have been previously designed targeting the acceptor splice sites [Wilton et al., 2007]. We decided to design and synthesize novel exon 16 and 26 AONs targeting exon-internal sequences. The wtAON against exon 33 is 5 nucleotides shorter than the one reported previously [Wilton et al., 2007]. AON on exon 34 was already designed and tested, but because it did not produce any skipping [Wilton et al., 2007], we designed a novel AON closer to the $3^{\prime}$ splice site.

ESE-finder software showed that the mutations in exon 10 and 34 did not result in differences in predicted SR protein binding sites. In contrast, the mutated exon 16 shows two additional SR proteins binding sites caused by the mutation (among these, the highest score was for SC35 protein). In exon 33, the mutation causes the formation of a binding site for the SC35 protein. The exon 26 mutation is responsible for the loss of two SR protein binding sites.

Taking these data into account and considering that our scope was to also include mutation-specific AONs, we were able to design msAONs for exons $10,16,26$, and 33 according to the rules described above (see Materials and Methods section). The mutation in exon 34 consists of the insertion of a novel stretch of 37 nucleotides, and we decided to design the msAON to target the inserted sequence. This stretch does not result in significant changes in SR protein binding sites as predicted by ESE-finder analysis.

\section{In Vitro Cell-Free Splicing Assays}

The five mutated exons $(10,16,26,33$, and 34 from patients 3 , $7,18,21$, and 23 in Table 1) were cloned into the pBG splicing vector [Gualandi et al., 2003] and analyzed using in vitro splicing assays. Splice variants were characterized and all the exon-exon junctions were sequenced (data not shown). In all cases the sequencing of the cloned splicing products demonstrated that the dystrophin mutated exons were correctly spliced between exons 2 and 3 of the rabbit $\beta$-globin gene (Fig. 1A). Antisense modulation using $2^{\prime} \mathrm{OMePS}$ msAONs and wtAONs was able to induce exon skipping in four out of five constructs in cell-free splicing assays at the scaled AONs concentration used (Fig. 1B). The semiquantitative method we adopted is based on the quantification of the amount of the spliced transcript (included exon) compared to the total amount of product (spliced and unspliced) in the presence of $A O N$ relative to the same ratio in the absence of $A O N$. In a major part of the in vitro assays the exon-skipping percentage did not depend on the AON concentration (Fig. 1C). We therefore calculated the average value of the skipping percentages (Fig. 1D), which could be more easily compared to the AONs efficacy in patient-derived cells. We observed that msAON was more efficient for skipping exon $34(85.55 \% \mathrm{msAON}$ vs. $9.19 \%$ wtAON $)$ and exon $10(25.63 \%$ msAON vs. $14.83 \%$ wtAON), whereas wtAON was more efficacious for exon 33 skipping $(50.72 \%$ wtAON vs. $19.93 \%$ msAON). For exon 16 we did not observe any differences in skipping efficacy between wt and msAON (about 5\%), whereas we were not able to skip exon 26 in vitro (Fig. $1 \mathrm{C}$ and D).

\section{Myogenic Cell AONs Transfection and Transcription Analysis}

The same wt- and ms-AONs tested in the in vitro splicing system were used in cell cultures. Using both AON types we were able to detect two products representing the unskipped and skipped transcripts by both primary (exons 16 and 33) and nested RT-PCR (exon 26 and 34) in patient-derived cell cultures (Fig. 2A). In contrast, no exon 10 skipping was observed in either primary nor nested PCR (Fig. 2A), in accordance with previously published results obtained with the universal AON [Wilton et al., 2007]. These experiments were highly reproducible.

RT-PCR of both untreated and treated cells did not show any unspecific splicing product, excluding the cells carrying the exon 10 mutation, which always showed a low level of physiological exon 9 skipping. This event has also been observed in untreated control muscle cells [Aartsma-Rus et al., 2005; Reiss and Rininsland, 1994].

To precisely quantify the specific level of exon skipping as well as to detect very low skipping amounts, we used an ESRA approach for all patient-derived treated and untreated cells. The 

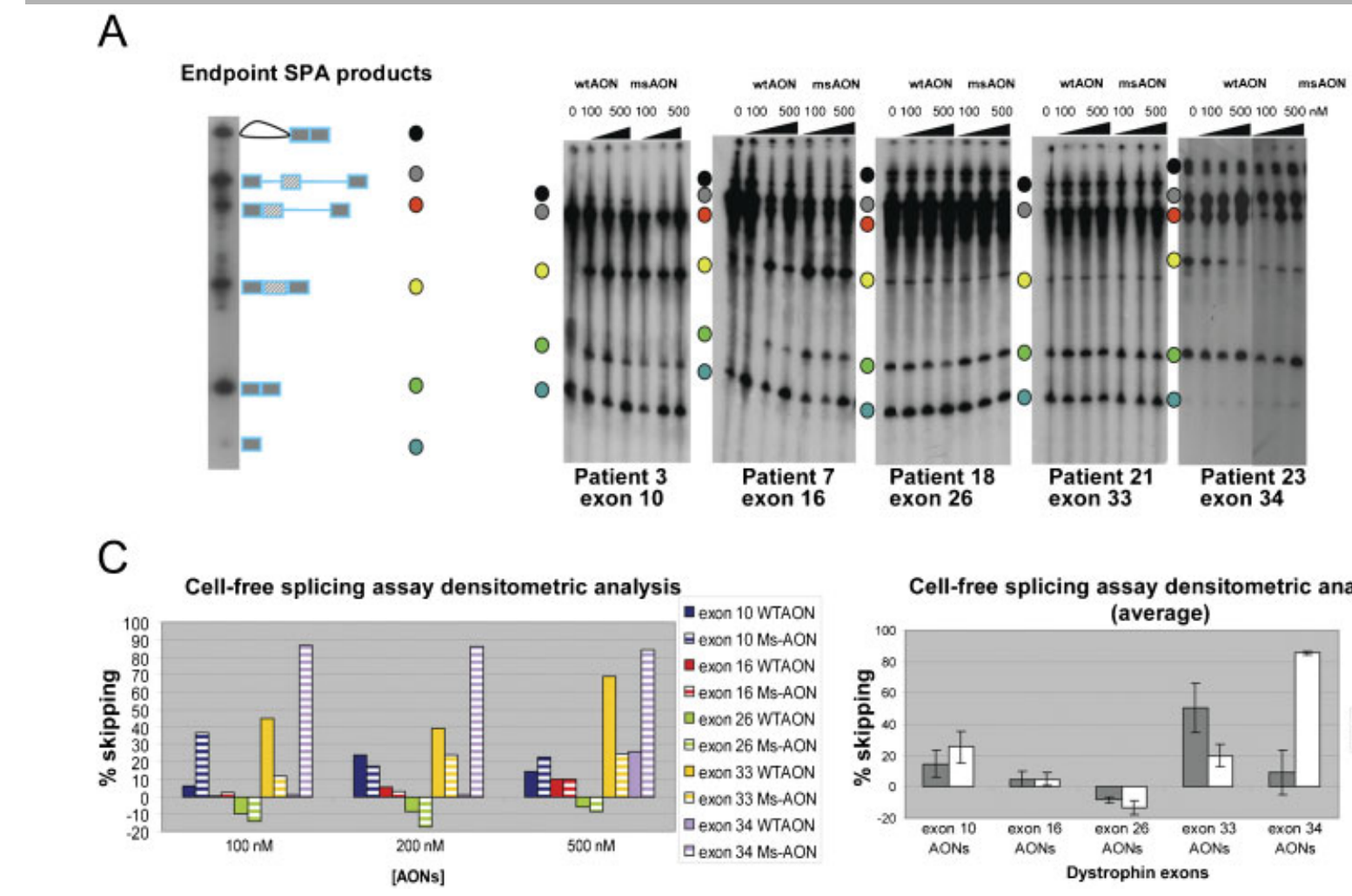

Figure 1. A: In vitro cell-free splicing assay endpoint (incubation time of 3 hours), with a scheme of all possible products. Colored dots represent the different products: black: lariat molecule; gray: unspliced template; red: splicing intermediate; yellow: exogenous exon spliced between $\beta$-globin exons 2 and 3; green: exogenous exon skipped transcript ( $\beta$-globin exons 2 and 3); blue: $\beta$-globin exon 2. B: Specific exon skipping using $2^{\prime} O M e P S A O N s$ in the in vitro cell-free splicing assays. wtAONs were designed on a wild-type sequence, msAONs were designed on mutated sequences. The five mutated exons (patients $3,7,18,21$, and 23) were in vitro spliced in the presence of scaled AONs concentrations $(100,200$, and $500 \mathrm{nM})$. C: Graphic representation of the densitometric analysis of the exon skipping percentages obtained using the 100,200, and $500 \mathrm{nM}$ concentrations of all the different A0Ns. The colors and bars differentiate the skipping efficacy of each mutated exon with wt or mSAONs. D: Graphic representation of the skipping average value considering the results obtained using all the scaled AONs concentration.

quantification was made using both adjacent dystrophin exons and $\beta$-actin as reference transcripts and in comparison to nontreated cells. The skipping percentages are shown in Fig. 2B. Comparing the $\mathrm{Ct}$ values obtained with proximal and distal dystrophin exons ( $\beta$-actin as reference), a $5^{\prime}-3^{\prime}$ transcriptional unbalance emerged, with $5^{\prime}$ transcripts more abundant in respect to the $3^{\prime}$ transcripts (data not shown). We therefore quantified the skipping percentage of the target exons using adjacent exons as references.

In patient 3 (c.1132_1135dup in exon 10) ESRA assay using exons 6 and 16 as references showed exon 10 skipping levels of $10.9 \%$ with msAONs, but only $2.4 \%$ of skipping with the wtAON. In contrast, patient 23 (c.4780delTins 37 in exon 34) revealed skipping of $6.5 \%$ with wtAON and only $1.6 \%$ with msAON, using exons 26 and 40 as references. For patients 7 (c.1912delC in exon 16), 18 (c.3447_3448delinsTT in exon 26) and 21 (c.4565delT in exon 33) exon skipping levels were comparable for wt and $\mathrm{ms}$ AONs. Exon 33 skipping levels were very high: 58.8\% (wtAON) and $58.3 \%$ (msAON) using exons 10 and 26 as references, exon 16 skipping levels were lower: $13.2 \%$ (wtAON) and $15.8 \%(\mathrm{msAON})$, using exons 10 and 20 as a reference, and skipping levels for exon 26 were $1.6 \%$ (wtAON) and $2.3 \%(\mathrm{msAON})$, with exon 10 and 16 as reference exons. ESRA analysis, assessing the skipping level through comparison with many references, provided robustness to the data, allowed us to detect the exon 10 skipping not visible by
RT-PCR, and identified a low level (less than $0.5 \%$ ) of spontaneous skipping of the mutated exons in all untreated cells.

In conclusion, the difference in skipping efficiency is significant for exons $10(p=0.0002)$ and for exon $34(p=0.0017)$, because msAON induced higher levels of exon 10 skipping, and wtAON was more efficient for skipping exon 34 (Fig. 2B). In contrast, the skipping efficiencies for exons 16 and 26 did not vary significantly.

\section{Immunofluorescence Analysis}

Immunofluorescence analysis of desmin (data not shown) and developmental myosin heavy chain (Fig. 3A) was performed to define the differentiation stage of the Myo-D transformed fibroblasts. Double-labeling with antidystrophin antibodies revealed the rescue of the protein in all patient-derived cells treated with wtAONs or msAONs. Blinded observation as to the treatment state was performed to quantify the cytoplasmic fluorescence counting fluorescent cells/number of nuclei. The number of dystrophin positive myotubes varied significantly from 5 to $15 \%$. Lowest levels (5\%) were observed for cells with the mutation in exon 10 and treated with the wtAON, for exon 26 treated with both wt and msAONs, and for exon 34 treated with msAON. This corresponds to the lower levels of exon skipping observed for these AONs (Fig. 2B). Dystrophin localized at the sarcolemma in all transfected myotubes (Fig. 3A and B). 


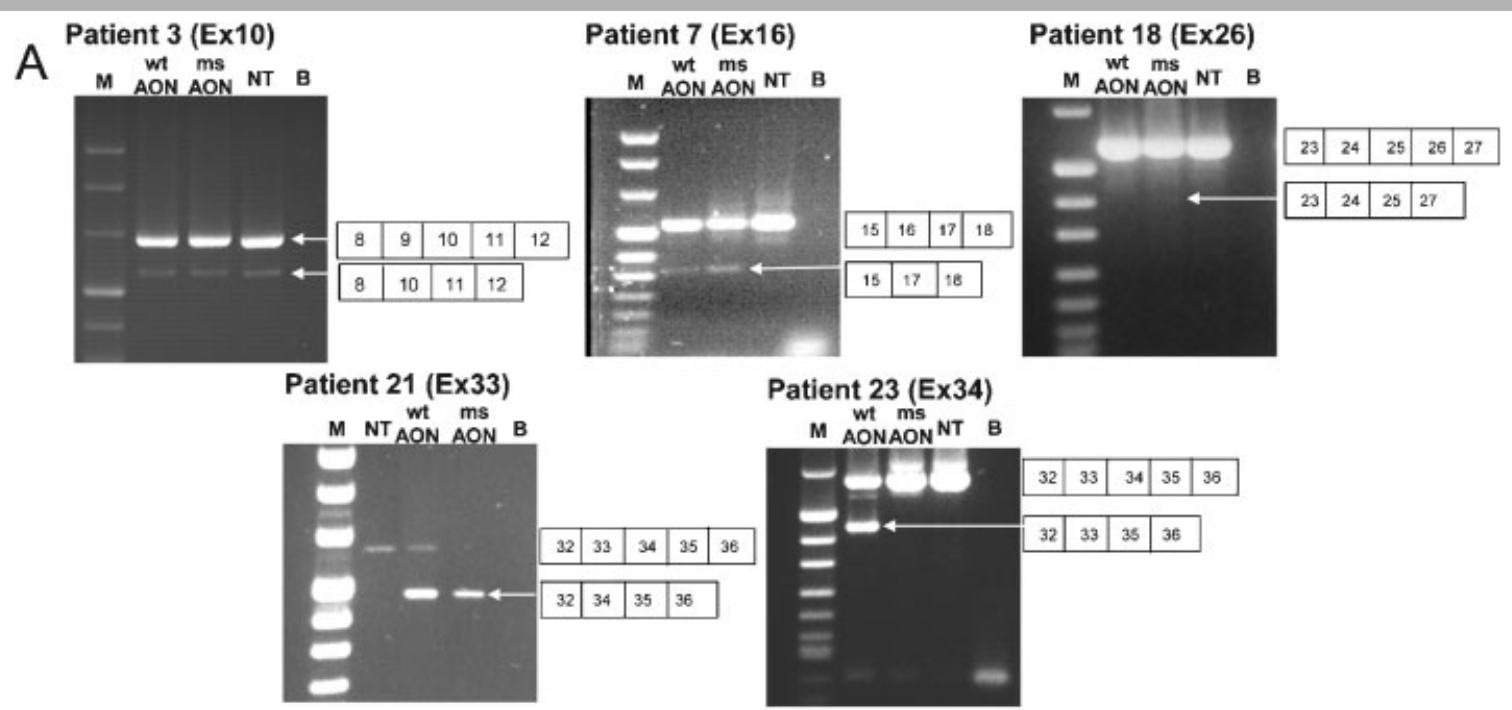

B

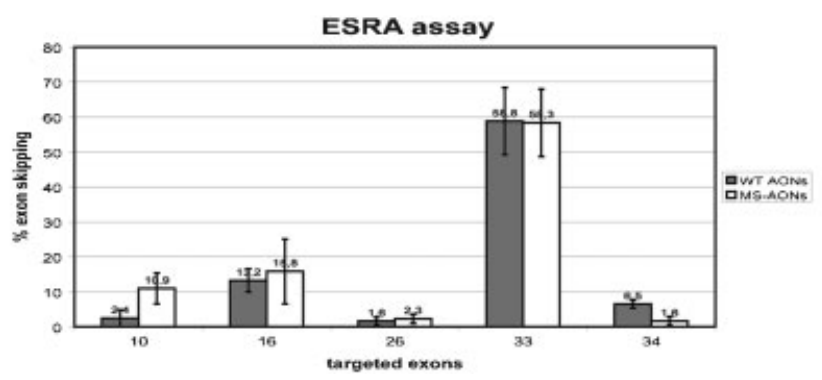

Figure 2. A: RT-PCRs of AONs-induced exon skipping in myogenic cells. Exons 10, 26, and 34 (patients 3, 18, and 23, respectively in Table 1) skipping was detected by nested RT-PCR, for the other two exons (30 and 33), primary RT-PCR only was performed. M is molecular weight marker VIII, B: blank sample. B: Graphic representation of dystrophin exons 10, 16, 26, 33, and 34 skipping quantitative differences in patientderived AONs-treated Myo-D transformed fibroblasts in comparison with untreated cells calculated by Real-time assay (ESRA). Histograms represent the percentage of specific exon skipping using either wtAONs (gray bars) or msAONs (white bars) of treated cells with respect to untreated cells.

\section{Discussion}

The aim of our study was to induce AON-mediated exon skipping of in-frame exons containing small mutations. We tested 2'OMePS AONs targeting both wild-type and mutated sequences to evaluate the skipping performance and to compare the skipping efficiency between ms and wtAONs. We took advantage from a large cohort of patients we had identified to carry small mutations. We modulated both in cell-free and in patient-derived cells 5 dystrophin "in frame" exons (10, 16, 26, 33, and 34) containing five different small DMD mutations.

For the five selected dystrophin exons, the splicing was effectively reproduced in cell-free assays; however, AONs modulation was successful only for exons $10,16,33$, and 34 , whereas exon 26 could not be skipped. For exons 10 and 34 msAONs worked better, whereas for exons 16 and 33 the wtAONs produced higher skipping levels. These data suggest that cell free splicing assays might be used to test the exon skipping propensity. However, because the exon composition/length as well as the flanking intronic regions are crucial for the in vitro splicing [Eperon et al., 1988], peculiar mutations like the long stretch included in the mutated exon 34 could hamper the optimal AONs identification in the in vitro assay. Therefore, it cannot be considered a general tool for optimizing the design of the AONs.

The fact that our test produced quite different results compared to patient-derived myogenic cells underlines this. For the mutated exons 16 and 33, we obtained similar levels of exon skipping with both wt and msAONs, comparable to what was previously described for wt exons [Wilton et al., 2007].

The mutation involving exon 10, not altering the splicing enhancer quality/distribution and therefore expected to be silent in term of skipping propensity, makes the mutated exon vulnerable to skipping with a $10.9 \%$ efficacy with msAON; slightly higher than described for the wt exon in myogenic cells testing [Wilton et al., 2007]. A possible explanation of this partially positive finding is that the mutation changes the exon 10 conformation. Although encouraging, the ESRA on exon 10 does not allow us to determine whether a contextual exon 9 skipping occurs [Wilton et al., 2007]. However, we underline that exon 9 skipping is a physiological, very common alternative splicing event, and it might therefore be very difficult to sort out its exclusion from the transcript. In addition, as exon 9 is in-frame, this exon skipping is unlikely to be detrimental.

Exon 26 was considered an exon difficult to skip (ratio $<10 \%$ ) [Wilton et al., 2007]. In our hands, both wtAON and msAON induced indeed a similar, very poor skipping level (1.6 and 2.3\%, respectively). This finding does not seem to reflect the expected increase of exon 26 skipping propensity linked to the loss of the two SR protein binding sites due to the mutation, as predicted by the ESE finder program.

The insertion in exon 34 is difficult to analyze. Although it appears not to have any modifying influence on splicing factors 


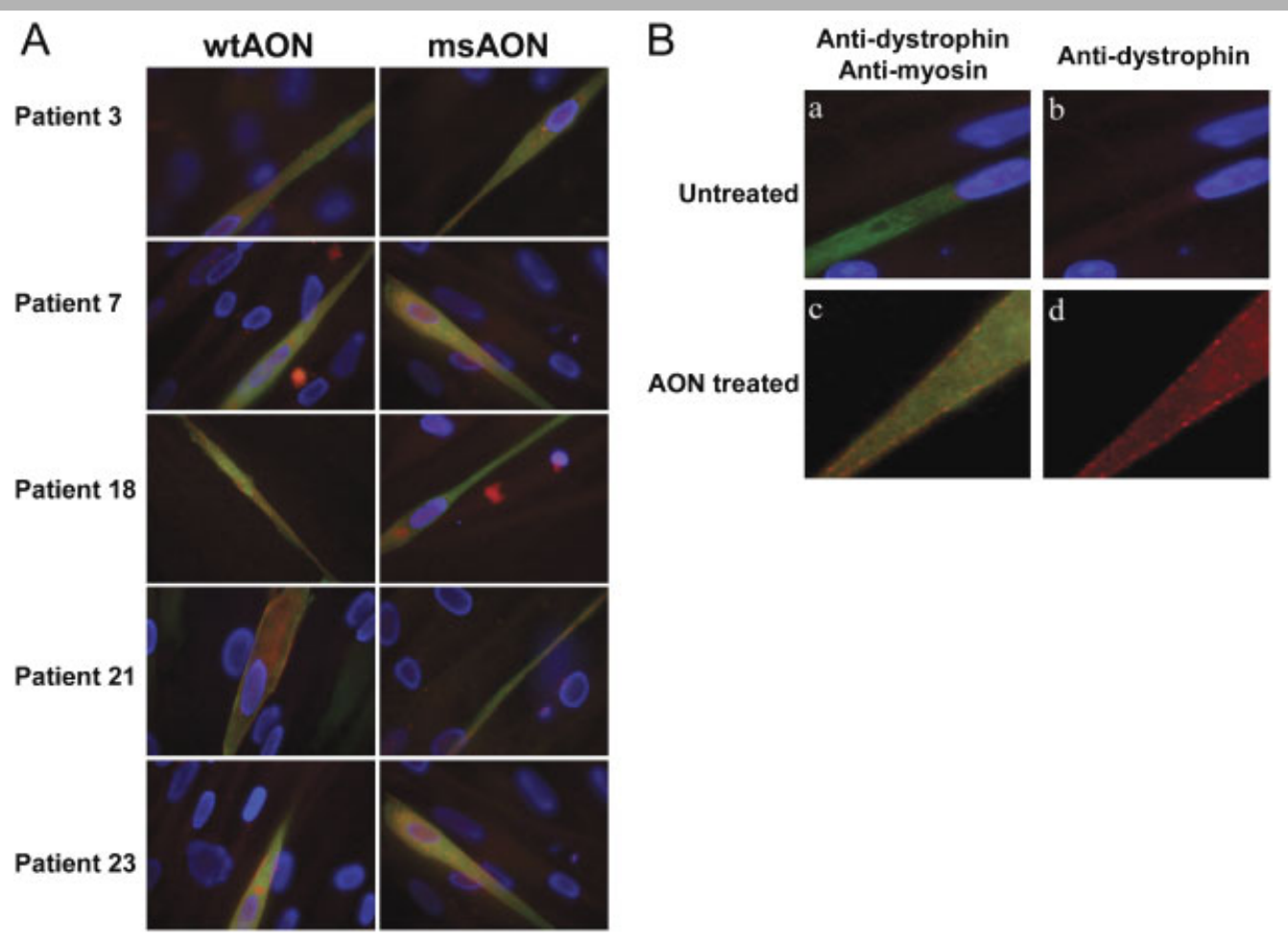

Figure 3. Immunofluorescence analysis of dystrophin in patients' cells. A: Double labeling with antimyosin heavy-chain (green) and dystrophin (red) antibodies clearly demonstrated the rescue of dystrophin expression in Myo-D tranformed fibroblasts of all treated patientderived cells. More than $50 \%$ of the AON-treated cells in all examined fields showed cytoplasmic green fluorescence, which was quantified as fluorescent cells/number of nuclei. Among these, 5-15\% myotubes were dystrophin positive. High magnification (B, d) demonstrates the correct protein localization at the sarcolemma. wtAON indicates antisense oligonucleotide designed on the wild-type exon, msAON indicates antisense oligonucleotide designed on the mutation. B: Untreated and AON treated patient's cells stained with antimyosin heavy-chain and antidystrophin antibodies. No dystrophin signal is detected in untreated cells $(a, b)$, whereas dystrophin membrane signal is observed upon the induced exon skipping in treated cells (c, d).

binding sites, obviously this long stretch may alter the exon skipping propensity in other ways. In fact, this mutation made the exon skipping propensity in myogenic cells increase, which is in contrast with previously published data [Wilton et al., 2007]. Indeed, we induced the skipping of the mutated exon 34 in $6.5 \%$ of the dystrophin transcript using the wtAON in patient-derived cells. A possible explanation is that the insertion/deletion mutation (insertion of 37 nucleotides and deletion of 1 nucleotide) enhances the exon 34 skipping propensity by enlarging the distance between motifs recognized by the SR proteins and other splicing proteins. Alternatively, the RNA secondary structure change could affect the recognition of the mutated exon, even though the accessibility of the splice sites is the same for wild-type and mutated exon 34 (data not shown).

In conclusion, we induced the specific exon skipping in four cellfree assays and in all five myogenic cells out of five selected mutated exons. When comparing the two different assays it was demonstrated that, in the absence of patient-derived cells availability, the cell-free assay could provide some information, but in vitro analysis in patient-derived cells is preferred. Furthermore, the results we obtained strongly suggest that some small mutations may unpredictably change the exon skipping propensity, implying that the exon skipping approach for in frame exons with small mutations deserves careful investigation. Furthermore, antisense targeting of small mutations might unravel splicing regulatory motifs, because flanking introns and exons are not involved in any rearrangement such as deletions/duplications and every cis-regulatory motif like intronic splicing enhancers (ISEs) or silencers (ISSs) can play a role in the exon defining process.

Notably, AON-mediated skipping of these five exons was never accompanied by other unspecific splicing products, such as the induction of cryptic splicing. The only alternatively spliced product was exon 9 skipping that was also observed in control myotubes. It is plausible (and very interesting) that fusion introns, resulting from deletion or duplication junctions, may influence adjacent exon recognition and therefore splicing accuracy.

Despite the promising results obtained using the exon skipping approach, another crucial point should be considered: the possible effect of the lack of skipped exons on the dystrophin protein. Isolated deletions of exons 10 and 34 are described causing a BMD or only high CK phenotype [Fokkema et al., 2005]. An isolated deletion of exon 16 causes high CK only [Schwartz et al., 2007; and personal observation]. For exons 26 and 33, however, no isolated deletions are reported in the Leiden Open Variation Database LOVD database [Fokkema et al., 2005; White and den Dunnen, 2006]. Therefore, the skipping of these exons may be of low predictive value on the resulting phenotype(s). It might also be possible that such deletions have not been identified yet due to the fact that these deletions do not result in any dystrophic phenotype. However, they all belong to the spectrin-like region (exon 10 to the repeat 1 , exon 16 to the repeat 3, exon 26 to the repeats $7-8$, exon 33 and 34 to the repeats 11 and 12), and, according to the literature data, their skipping is expected to have mild/BMD-like consequences on the protein function. 
The data presented here report the first attempt to modulate small dystrophin mutations by using both wild-type and "mutation-specific" AONs, and highlight both the peculiar splicing characteristics of mutated exons and the complexity of designing optimal AONs for exon skipping therapy toward personalized therapy.

\section{Acknowledgments}

The Telethon Italy Grants GGP05115 and GUP07011 (both to A.F.) are acknowledged. Thanks are also due to the TREAT-NMD Network of Excellence of EU FP6 No. 036825 (to L.M. and Telethon-Italy), to the NMD-CHIP EU FP7 Grant No. 223026 (to A.F.), to the Industria Chimica Emiliana (ICE Reggio Emilia, Italy) (to A.F.), and to Duchenne Parent Project Italy (to A.F.). We wish to thank Dr. Annemieke Aartsma-Rus (LUMC, Leiden, NL) for the helpful suggestions and critical reading of the manuscript. We would like to acknowledge Peter Taschner for helping with mutation nomenclature and annotation. Miss Monika and Henk van Zurksum, as well as Jolieke van Oosterwijk, have kindly revised the English language. The AONs used in this article have been patented at the University of Ferrara, Industrial Liaison Office, patent IP number TO2009A000487.

\section{References}

Aartsma-Rus A, De Winter CL, Janson AA, Kaman WE, Van Ommen GJ, Den Dunnen JT, Van Deutekom JC. 2005. Functional analysis of 114 exoninternal AONs for targeted DMD exon skipping: indication for steric hindrance of SR protein binding sites. Oligonucleotides 15:284-297.

Aartsma-Rus A, Fokkema I, Verschuuren J, Ginjaar I, van Deutekom J, van Ommen GJ, den Dunnen JT. 2009.Theoretic applicability of antisense-mediated exon skipping for Duchenne muscular dystrophy mutations. Hum Mutat 30:293-299.

Aartsma-Rus A, Janson AA, Kaman WE, Bremmer-Bout M, den Dunnen JT, Baas F, van Ommen GJ, van Deutekom JC. 2003. Therapeutic antisense-induced exon skipping in cultured muscle cells from six different DMD patients. Hum Mol Genet 12:907-914

Aartsma-Rus A, Janson AA, Kaman WE, Bremmer-Bout M, van Ommen GJ, den Dunnen JT, van Deutekom JC. 2004. Antisense-induced multiexon skipping for Duchenne muscular dystrophy makes more sense. Am J Hum Genet 74:83-92.

Aartsma-Rus A, Van Deutekom JC, Fokkema IF, Van Ommen GJ, Den Dunnen JT. 2006. Entries in the Leiden Duchenne muscular dystrophy mutation database: an overview of mutation types and paradoxical cases that confirm the readingframe rule. Muscle Nerve 34:135-144.

Aartsma-Rus A, van Vliet L, Hirschi M, Janson AA, Heemskerk H, de Winter CL, de Kimpe S, van Deutekom JC, 't Hoen PA, van Ommen GJ. 2008. Guidelines for antisense oligonucleotide design and insight into splice-modulating mechanisms. Mol Ther 9:105

Bennett RR, den Dunnen J, O'Brien KF, Darras BT, Kunkel LM. 2001. Detection of mutations in the dystrophin gene via automated DHPLC screening and direct sequencing. BMC Genet 2:17.

Buzin CH, Feng J, Yan J, Scaringe W, Liu Q, den Dunnen J, Mendell JR, Sommer SS. 2005. Mutation rates in the dystrophin gene: a hotspot of mutation at a CpG dinucleotide. Hum Mutat 25:177-188.

Cartegni L, Wang J, Zhu Z, Zhang MQ, Krainer AR. 2003 ESEfinder: a web resource to identify exonic splicing enhancers. Nucleic Acids Res 31:3568-3571.

Deburgrave N, Daoud F, Llense S, Barbot JC, Récan D, Peccate C, Burghes AH, Béroud C, Garcia L, Kaplan JC, Chelly J, Leturcq F. 2007. Protein- and mRNAbased phenotype-genotype correlations in DMD/BMD with point mutations and molecular basis for BMD with nonsense and frameshift mutations in the DMD gene. Hum Mutat 28:183-195.

Eperon LP, Graham IR, Griffiths AD, Eperon IC. 1988. Effects of RNA secondary structure on alternative splicing of pre-mRNA: is folding limited to a region behind the transcribing RNA polymerase? Cell 54:393-401.

Flanigan KM, von Niederhausern A, Dunn DM, Alder J, Mendell JR,Weiss RB. 2003. Rapid direct sequence analysis of the dystrophin gene. Am J Hum Genet 72:931-939.

Fokkema IF, den Dunnen JT, Taschner PE. 2005. LOVD: easy creation of a locusspecific sequence variation database using an "LSDB-in-a-box" approach. Hum Mutat 26:63-68.
Gualandi F, Rimessi P, Cardazzo B, Toffolatti L, Dunckley MG, Calzolari E, Patarnello T, Muntoni F, Ferlini A. 2003. Genomic definition of a pure intronic dystrophin deletion responsible for an XLDC splicing mutation: in vitro mimicking and antisense modulation of the splicing abnormality. Gene 311:25-33.

Havenga MJ, Lemckert AA, Ophorst OJ, van Meijer M, Germeraad WT, Grimbergen J, van Den Doel MA, Vogels R, van Deutekom J, Janson AA, de Bruijn JD, Uytdehaag F, Quax PH, Logtenberg T, Mehtali M, Bout A. 2002. Exploiting the natural diversity in adenovirus tropism for therapy and prevention of disease. J Virol 76:4612-4620.

Hoffman EP, Brown Jr RH, Kunkel LM. 1987. Dystrophin: the protein product of the Duchenne muscular dystrophy locus. Cell 51:919-928.

Hofstra RM, Mulder IM, Vossen R, de Koning-Gans PA, Kraak M, Ginjaar IB, van der Hout AH, Bakker E, Buys CH, van Ommen GJ, van Essen AJ, den Dunnen JT. 2004. DGGE-based whole-gene mutation scanning of the dystrophin gene in Duchenne and Becker muscular dystrophy patients. Hum Mutat 23:57-66.

Koenig M, Beggs AH, Moyer M, Scherpf S, Heindrich K, Bettecken T, Meng G, Müller CR, Lindlöf M, Kaariainen H. 1989. The molecular basis for Duchenne versus Becker muscular dystrophy: correlation of severity with type of deletion. Am J Hum Genet 45:498-506.

Monaco AP. 1989. Dystrophin, the protein product of the Duchenne/Becker muscular dystrophy gene. Trends Biochem Sci 14:412-415.

Nielsen KB, Sørensen S, Cartegni L, Corydon TJ, Doktor TK, Schroeder LD, Reinert LS, Elpeleg O, Krainer AR, Gregersen N, Kjems J, Andresen BS. 2007. Seemingly neutral polymorphic variants may confer immunity to splicinginactivating mutations: a synonymous SNP in exon 5 of MCAD protects from deleterious mutations in a flanking exonic splicing enhancer. Am J Hum Genet 80:416-432.

Reiss J, Rininsland F. 1994. An explanation for the constitutive exon 9 cassette splicing of the DMD gene. Hum Mol Genet 3:295-298.

Rimessi P, Sabatelli P, Fabris M, Braghetta P, Bassi E, Spitali P, Vattemi G, Tomelleri G, Mari L, Perrone D, Medici A, Neri M, Bovolenta M, Martoni E, Maraldi NM, Gualandi F, Merlini L, Ballestri M, Tondelli L, Sparnacci K, Bonaldo P, Caputo A, Laus M, Ferlini A. 2009. Cationic PMMA nanoparticles bind and deliver antisense oligoribonucleotides allowing restoration of dystrophin expression in the $\mathrm{mdx}$ Mouse. Mol Ther 17:820-827.

Roberts RG, Gardner RJ, Bobrow M. 1994. Searching for the 1 in 2,400,000: a review of dystrophin gene point mutations. Hum Mutat 4:1-11.

Roberts RG, Passos-Bueno MR, Bobrow M, Vainzof M, Zatz M. 1993. Point mutation in a Becker muscular dystrophy patient. Hum Mol Genet 2:75-77.

Roest PA, van der Tuijn AC, Ginjaar HB, Hoeben RC, Hoger-Vorst FB, Bakker E, den Dunnen JT, van Ommen GJ. 1996. Application of in vitro Myodifferentiation of non-muscle cells to enhance gene expression and facilitate analysis of muscle proteins. Neuromuscul Disord 6:195-202.

Schwartz M, Dunø M, Palle AL, Krag T, Vissing J. 2007. Deletion of exon 16 of the dystrophin gene is not associated with disease. Hum Mutat 28:205.

Solis AS, Shariat N, Patton JG. 2008. Splicing fidelity, enhancers, and disease. Front Biosci 13:1926-1942.

Tuffery-Giraud S, Saquet C, Chambert S, Echenne B, Marie Cuisset J, Rivier F, Cossee M, Philippe C, Monnier N, Bieth E, Recan D, Voelckel MA, Perelman S, Lambert JC, Malcolm S, Claustres M. 2004. The role of muscle biopsy in analysis of the dystrophin gene in Duchenne muscular dystrophy: experience of a national referral centre. Neuromuscul Disord 14:650-658.

van Deutekom JC, Janson AA, Ginjaar IB, Frankhuizen WS, Aartsma-Rus A, Bremmer-Bout M, den Dunnen JT, Koop K, van der Kooi AJ, Goemans NM, de Kimpe SJ, Ekhart PF, Venneker EH, Platenburg GJ, Verschuuren JJ, van Ommen GJ. 2007. Local dystrophin restoration with antisense oligonucleotide PRO051. N Engl J Med 357:2677-2686.

Welch EM, Barton ER, Zhuo J, Tomizawa Y, Friesen WJ, Trifillis P, Paushkin S, Patel M, Trotta CR, Hwang S, Wilde RG, Karp G, Takasugi J, Chen G, Jones S, Ren H, Moon YC, Corson D, Turpoff AA, Campbell JA, Conn MM, Khan A, Almstead NG, Hedrick J, Mollin A, Risher N, Weetall M, Yeh S, Branstrom AA, Colacino JM, Babiak J, Ju WD, Hirawat S, Northcutt VJ, Miller LL, Spatrick P, He F, Kawana M, Feng H, Jacobson A, Peltz SW, Sweeney HL. 2007. PTC124 targets genetic disorders caused by nonsense mutations. Nature 447:87-91.

White SJ, den Dunnen JT. 2006. Copy number variation in the genome; the human $D M D$ gene as an example. Cytogenet Genome Res 115:240-246.

Whittock NV, Roberts RG, Mathew CG, Abbs SJ. 1997. Dystrophin point mutation screening using a multiplexed protein truncation test. Genet Test 1:115-123.

Wilton SD, Fall AM, Harding PL, McClorey G, Coleman C, Fletcher S. 2007. Antisense oligonucleotide-induced exon skipping across the human dystrophin gene transcript. Mol Ther 15:1288-1296. 